\title{
III. Cortisona en la enfermedad reumática
}

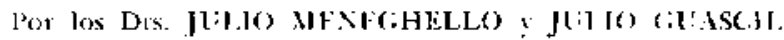

El uso del ACTH y cortisunis en la enfermedad remmitica iniciado por Hench en 1919, abrió uni meva etapa en el tratamiento cle csta afecrión, que es sin cluda, la más impor. tante enure las cnfermedades del mescnquima, que caen en la eslera de acción de estas hormonas.

Aparecen entonces justificados los numerosos ensayos terapjétiticos, que han tratado de delimitar los verdaderos alcances de este nue. vo tratamiesto.

La acción protectora de la cortisona sobre el fenómeno inllamatorio tisular, interfiriendo en el mecanismo ácido hialurónico-hialuronida:a, permite suponer gue en la inllamación reumática actuaría acortanklo la tase exudativa, con lo que se reclucirían los procesos proliferativos y de repararión, haciéndose mínimo el daîn residual.

Desde el punto de vista de lat experimentación clínica, se hat planteado los interrogantes de si estas hormonas son capaces de reducit la evolución natural de la enfermedad reumitica, si previenen el daño cardiaco y si actúan favorablemente sobre lit carditis, lográndosc evitar Tas lesiones cardiatcos delinitivas. Conviene clesde ya insistir en las dilicultades que se presentan al interpretar la elicacia de un tratamiento en esta ilfeción tam proteiforme en su sintomatologia y cvolución, de la cual se ha dicho que se determina por sí misma.

Expondremos primeramente algunas informariones obteridas cn la literatura extranje- ra, parie teferimos después a muesia experionria hasida en el tratamiento con cortisona de nun ge upo de niños reumáticos.

Hench y cols. (1950) emplean ic: $\mathrm{TH}$ y cortisonat en la eufermedad reumbition angda, observando remisión rípida de la liebre. poliax. tritis y taquicardia, alommando valores normales la sedimentación en un playo aproximado de 18 dias; observan inactivación de lit raditis, en plianos variables, aconsejandts un tratamiento prolongado, con dosis de ataque seguida de una dosis de mantención.

Matssel y Warten (1950) sc relieren at 20 (asos de enfermedad reumátical, tratados con ACTH, la mayoría de ellos con intersal cardi. is. Observan también la remisión rápida de la fiebre y poliartritis y en 5 cisos de carditis recientes, desaparecen los soplos en lapsos valriables entre algunas semanas y meses. Este último lececho, unido a la regresión de la insufi. ciencia cardítcil y signos de pericarditis observados en un grupo de enlemos, les pennite suporicr efectos favorables sobre da carditis. Resumen su impresión diciendo, que el proceso se acorta, se suprinte ha carditis attiva y has razones para suponer que la administración preco\% de la dhoga, evita el daño cardíaco.

Wilson y Iflplper (195]) conumican optimistats resuliados con el uso de ACTH, en 11 pacientes reusuáticos, 6 de los clales hacím cl primer brote de $1_{\text {a }}$ enfermedad. Se demuestra desaparicion rápida de la carditis, entro el 39 y $7^{\circ}$ díil de tratamiento, con ruración 
tompleta en los casus cu que no existía previo daño valvular. Señalan los autores lat jmporcaltcia del tratimiento precoz.

Barnes y cols. (1951) retieren tambien clectos favorabiles en 6 ele 10 casos tratides en lil primera crisis, clentostrando indemnidad cardiaca el control parcicado 1-10 mescs alespucs de abandonar el hospital; atribuyen al ACiH y cortisona la capacidad cle suprimir las manlestaciones agudas, insistiendo en la importan(ia del tratamiento precos.

Dorfinas y Smull (1951) relieren su expexiencia basada en ei tratamiento con s.'ll de ga niños reumáticos con evilente daño cardiaco. Demostraron efertos favorables solbre la cardilis, ya que remitieron la taquicardia, lrotes pericardiacos, signos de insuficiencia cardíina, en lo de l:3 enfernos y disminuyó la siluetil en 8 de 17 casos, durante el tratauiento. Sin enbargo, se observaron escasas variaciones en los soplos y no se previno lá muerte en casos de pancatditis. Su cxperienciat no les permite deducir que se modilique el curso normat de la afeccioin ni que sc prevengan las lesiones valvulates.

Resultados menos hatagadores comunican kuttner y cols. (1952) en el tratamiento con IC:TH y cortisona en un grupo de 18 reumíticos, 12 de los cuales correspondian al primer brote; en escos liltimos, se observó la persistencia de soplos ongánicos en todos los rasos y en 11 de ellos persistiós el agrandamiento cardíaco. No lus: evirlente según concluyen los autoces, que el trataniento disminuyera la duración de la carditis o influenciara el daño carsliaco.

En Lruguay, el tratamien:o de un importante número de casos, permite comprobar a Guerra (1954) el efecto favorable de la cortisonil, en las carditis reumáticas de primer bro te, con desaparición de los soplos en 2 a 3 semanas, siendo nulos los efectos en las formas malignas.

Nos parce de interis agregar lia impresión actuil acerca de la eficacia de estas hormonas en la enfermedad reumática, sintetizada por Honch (1952) en un análisis de los alcances terapćticos de estas drogas en diferentes afecciones col las cuales es útil su empleo. Se les ccconoce una acción favorable sobre las manifestacioncs agudas del proceso: liebre, poliartritis, taquicardia, toxemia; se normalizan en pocas semanas la sedimentación y alteraciones electrocarcliograficas. En ciejtos casos de carditis aguda los signos se han ateluado a desaparecido rápidamente. Sin embargo, aunque estas drogas suprimen sintomis, no curan la enfermedad reumálica ni está aún claramente estalbkcido que acorten la durarión matural de la fase aguda; pero existe alguna evidenciá de que pueden suprimir las lases exudativa y pro. liferativa de: la carditis reumátici aguda, con lo cual se podria evitar gran prate del daño cardíaco.

\section{Nuestra experiencia}

Está basada en la observación de utl g'upo de niños reumiticos tratados com cortisona en el curso de este año. Es un inaterial 110 seleccionado que corresponde a los ingresos it una Sección de Medicina del Hospital. ios referiremos solo a 22 casos que tienen un perfodo de observación mayor, pero anticipamos que nuestro estudio es sólo una comunicatción prelininat, yá que conclusiones más definidas sólo se pueden obtener después de un control prolongado de los enfernos.

La edad de los pacientes osciló entre 5 y 12 aīos, correspondientlo 16 casos a ingresos durante la primera crisis. La gravedad del daño cardíaco presentc en 21 enferoos, fuć estimada leve en 3 rasos y grape en los 19 restantes.

Los enfermos fueron sometidos a un plan de tratamiento, que incluia las medidas labituales de reposo, rćgimen dietćtico, suplemen. tos vitamínicos, aroliotónicos y diuréticos en lus casos de descompensación carclácáa lat cor. tisona fué administrada por via intranusculat. de acuerdo a un esquema arbitiario, que consistia en el suministro de una dosis de 100 mgrs. los tres primeros días, en formal fraccionada, 50 mgrs. Ios tres isguientes y 25 urgrs. cliarios hasta completar un mes de tratamiento.

Se practicó una observación detallada de lit evolución clinica, verificándose sistentíticamente eritrosedimentación, electrocardiograma, estudio radiológico, controles cle uremia y glicemia, hemogtama y anílisis de orinat.

\section{Resultados}

Nuestros entermos no presentatron con frecuencia temperatura elcvada ni manilestacionei atticulares, pero en los casos en que cstos signos eran ostensibles, asistimos il su rápida rimisión en los primeros 4 dias de tratamiento. Fin el curso de las 2 primeras vemanas observamos en la nayoria de los pacientes mejoria manitiesta del estado general, incluyendo la res nperación del apetitu.

I a normalización de la sedimentatión se al. canz en plazos rariables entre 10 y 45 dias, reciticindose en la mayoría de los pacientes 
en las 1 semanas consecutivas a la iniciación Jel tratamiento (grifico 1).

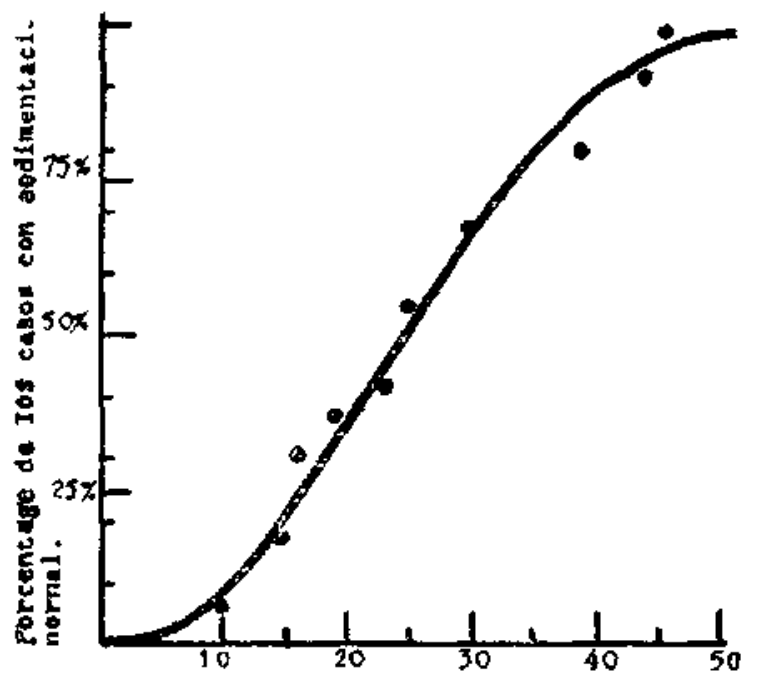

Dias

Gráfico $\mathrm{N} 1$

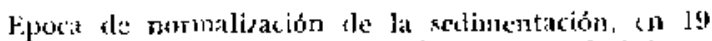
casos de enfermedad reuntilica, durante la adininisorilcion de cortisnana.

Ln 19 casos se consiguió la inactivación de la carditis, efectuándose en 11 durante el periodo de tratamiento. Se observó cierta relación entre el momento de iniciación del tratamiento y la duración del período de inactivación, que aparece más breve en los casos tratados más precozmente (gráfico 2).

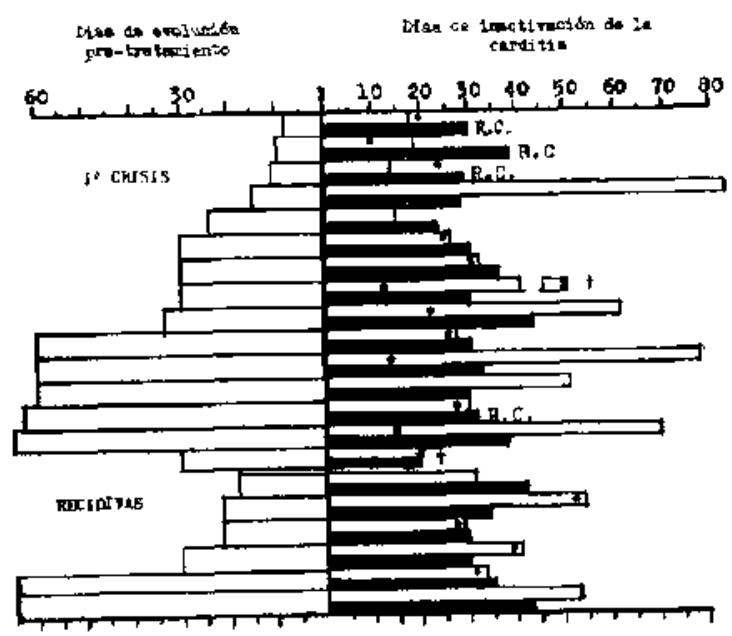

Gráfico No ?

Exolución die las manifestaciones fit actividad reumatica después kel tratamicnto con cortisona.
La evolución de los signos de carditis demostró mayar precocidad en la desaparición (4 primeras semanas) de la taquicardia, apagamiento de los rnidos. 3.er ruido, frotes y derrame pericardiaco, siendo variable la desapación del agrandamiento e insuficiencia cardiacas.

En 10 pacientes tratados en su primer ataque, persistieron soplos de naturaleza orgánica y en 4 de 9 casos con agrandamiento cardiaco persistió este signo después de la inactivación.

Se obtuvo curación sin secuelas en 1 enfermos, correspondiendo $\$$ de ellos a formas leves de carditis en los cuales se inició el tratamiento con cortisona en los primeros 15 dias te enfermetlad. En cambio el otro era una pancarditis remmática, tratada después de 2 meses de evolución, en la cual se consiguió la inclemnidad cardíaca 83 días después de $1 a$ iniciación de la terapia hormonal.

in los 6 casos de recurrencias, el estado cardíaco despues de la inactivación demostró en un caso, agrandaniento cardíaco que anteriormente no existia y en otro se agregó una nueva lesión valvular.

Dos pacientes fallecieron, presentando ambos formas graves de carditis con signos de descompensación. En uno de ellos la muerte se produjo durante el tratamiento después de haber experimentado mejoría transitoria y en el otro algunos días después de suspendida la cortisona en una recaida relacionada con una amiglalitis aguda intcrurrente.

Fntre los elecios secundatios de la droga, observamos edema en 9 casos, hipertricosis en 2 y acné en un caso. Fuc frecueute la alteración de la fisononia denominada "cara de luna". En un enfermo con carditis grave y signos de insuficiencia cardiaca congestiva, vimos agravarse lit sintomatologia lasta el punto que fué necesaris suspender transitoriamente la droga, pero con la reiniciación del tratamiento asislimos a la inactivación del proceso.

Como lo scñilamos anteriormente, esta revisión esquemítica que hemos expuesto de la evolución de un grupo de niños reumáticos tratados con cortisona, es sólo una comunicación preliminar. Estamos en la acturalidad ampliando nuestra casuistica y controlando la evolución de los casos tratados, para fundamentar mejor nuestrat experiencia como un aporte más a este tema, que tan justificadas incuieudes ha provocado en quienes buscan la solución del grave problem qu que significa la enfermedad reumática en el niño. 


\section{Sumatio}

Se revisa bibliografía acerca del tratimiento le la cnfexmedad reumática con $\Lambda C T H$ y cortisona.

Se exponen los resultados obtenidos en 2\% niños reumíticos tratados con cortisoná, 16 de los cuales liacian el primer brote de la enfermexlad. En 19 casos se consignó compromiso cardíaco grave.

La droga fuc administrada en rlosis arbitraria inicial de 100 mgrs. los 3 primeros slías, en forma fraccionada, $50 \mathrm{mgrs}$. los $3 \mathrm{si}$ guientes y 25 mgrs. hasta completar un mes de tratamiento.

La remisión de $\mathbf{l}_{\mathbf{a}}$ temperatura y signos articulares se efectuó en los 4 primeros dias de tratamiento, observándose mejoría general en el curso de las 2 semanas consecutivas a la iniciacicin de lia terapia hormonal.

La sedimentación se normalizó en la mayoria de Ios pacientes en las 4 primeras scmamas de tratamiento.

En 19 casos se inactivó la carditis, electuán- dose en 11, durante la aclministración de la droga. El periodo de inactivación aparecia en general más breve en los casos tratados más precozmente.

Se obtuvo curación sin secuelas en 1 enfermos, 3 de las cuales eran formas leves de carditis Lratadas durante el primer brote. El restante correspondia a una pancarditis reumática tratada después de dos meses de evolución.

Entre 6 pacientes tratados durante una recurrencia, se observó en 2 accentuación del da. no cardíaco.

Dos enfermos, con formas graves de enfermedad reumática fallecieron, uno durante la administración de la droga, y el otro clespués de ипа aparente inactivación, seguida de grave recrudecencia del proceso.

Consideramos aún breve el período de observación de los enfermos para obtener conclusiones definidas. Sin embargo, la cortisona nos parece una droga de utilidad en el control de la actividad reumática, especialmente cuando su administración se efectúa precozmente. 